\title{
How a Swirling Tail Helps Microbes Swim
}

\author{
A microswimmer that rotates its body and tail in opposite directions can \\ propel itself in elastic, non-Newtonian fluids.
}

\section{By Erika K. Carlson}

ike human swimmers, microorganisms employ various techniques to propel themselves through fluids. But the fluids that these microswimmers navigate, such as mucus in the human body, are often complex and non-Newtonian, enabling strokes that wouldn't work in a swimming pool. Now, Jeremy Binagia and Eric Shaqfeh of Stanford University have analyzed one such technique that works in complex, elastic fluids [1].

The researchers investigated a model swimmer made up of two differently sized spheres. Previous work had studied how a microswimmer of this shape would move through fluid when its swimming technique was driven by an external torque applied, for example, via a magnetic field. But Binagia and Shaqfeh looked at a mechanism that relied on no external torques. In their system, one sphere rotated itself in one direction, while the other rotated in the opposite direction-similar to the way in which an E. coli bacterium rotates its body one way and swirls a bundle of flagella the other way.
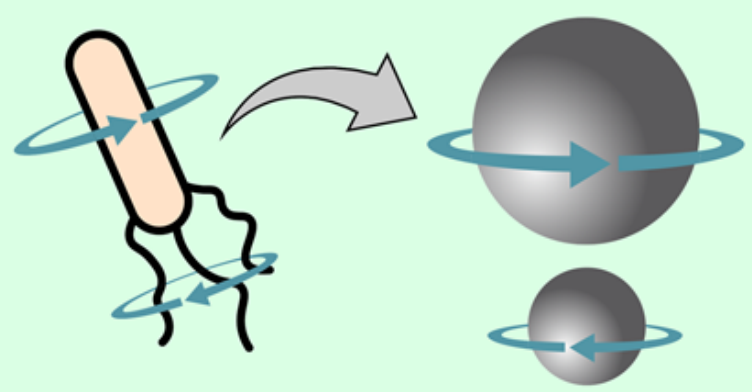

Credit: J. Binagia/Stanford University
With a combination of analytical calculations and numerical simulations, the researchers determined that their swirling microswimmer would propel itself in the direction of the larger sphere when placed in an elastic fluid, even though this swimming technique would not work in a Newtonian fluid. They also analyzed the dependence of the microswimmer's swimming speed on factors such as the elasticity of the fluid and the relative sizes of the spheres, and they found good agreement between their predictions and experimental measurements of $E$. coli. The researchers say that understanding such swimming methods in complex fluids could have biomedical applications, such as propelling microswimmers to deliver drugs in a targeted way.

Erika K. Carlson is a Corresponding Editor for Physics based in New York City.

\section{REFERENCES}

1. J. Binagia and E. S. G. Shaqfeh, "Self-propulsion of a freely suspended swimmer by a swirling tail in a viscoelastic fluid," Phys. Rev. Fluids 6, 053301 (2021). 